\title{
Vedo non vedo la (il) bralette
}

\author{
Miriam Di Carlo
}

PUBBLICATO: 31 MARZO 2019

La parola bralette (pronuncia /bra'lıt/), indica un tipo di reggiseno senza imbottitura, coppa o ferretto con ampi triangoli che coprono il seno e diventano spalline. Si tratta dunque di un reggiseno non preformato, che di solito presenta una piccola fascia sotto il seno, spesso decorato con pizzi e merletti. Dalle descrizioni che se ne ricavano sul web, questa tipologia di reggiseno esisteva già all'inizio del Novecento pur non avendo una parola specifica che la designasse. La moda degli ultimi anni ha rilanciato il modello in chiave contemporanea, associandogli la parola bralette, che ha avuto successo sulla scia di quello ottenuto dal prodotto.

A dispetto di quanto la forma, dotata del suffisso -ette tipico del francese, farebbe pensare, bralette è di origine inglese. Nell'Oxford English Dictionary (OED) è registrato (anche nella forma bralet/bra'le/) con due significati, uno raro e uno più attuale. Almeno fino agli anni ' 60 , bralette indica un tipo di indumento intimo che combina un reggiseno senza spalline a un corsetto, mentre, dagli anni '7o circa, designa l'oggetto cui facciamo riferimento oggi, ovvero un reggiseno morbido senza supporti, come ad esempio imbottiture o ferretti, disegnato per essere infilato dalla testa. Sempre secondo l'OED, oggi la parola può indicare anche un indumento femminile non necessariamente intimo, indossato spesso come un top (gli esempi riportati dall'OED per questa seconda accezione arrivano fino agli anni più recenti, ovvero al 2015).

Per quanto riguarda l'etimologia, la parola deriva da bra 'reggiseno', a sua volta dal francese brassière, con l'aggiunta del suffisso, sempre di origine francese, -let (anche -lette) che, secondo l'OED, in aggiunta a parole indicanti parti del corpo designa ornamenti che riguardano quella stessa parte del corpo: armlet ('braccialetto' daarm 'braccio'), frontlet ('sorta di tiara che scende sulla fronte' da front 'fronte'), ecc. Può aver senz'altro favorito la formazione della parola bralette la presenza, nel lessico inglese, di alcune parole di origine francese con cui si indicano capi d'abbigliamento intimo: anzitutto corselet (corslet), che dagli inizi XX secolo ha cominciato a designare un capo d'abbigliamento intimo modellato sugli antichi corsetti, e poi balconette, parola entrata nel lessico inglese a partire dagli anni ' 50 per indicare un corsetto con reggiseno a balconcino.

La parola bralette arriva nel lessico italiano come prestito non adattato e compare per la prima volta nel zorI, sul quotidiano in rete "Libero":

I reggiseni nella versione bralette o più costruita, modellano le forme perfettamente ([s.f.], Moda: Armani e Rihanna insieme per una capsule collection, liberoquotidiano.it, 23/II/20II).

Questa attestazione, in cui il termine è legato all'ambito specialistico della moda intima, rappresenta un caso isolato. A partire dal 20I3, la parola ricompare in testi in lingua italiana, per esempio sui social network:

Lingerie of the week: La Perla 'Baronessa' bralette (tweet di @Lingerie_4us, 28/3/2013).

Il risveglio della bralette: Ciao girls! Sono tornata da tre giorni dalla mia casa di Miami per un soggio... (tweet di @theAgencyRoma, 30/5/2013).

Una nuova ondata di occorrenze sul web si registra a partire dal 20r4:

In un'immagine, Rita indossa un bralette in rete nera (Jennifer Chan, Rita Ora in topless per Elle U.K., eonline.com, 8/4/2014).

In casa porto una bralette di mais bianca (Iо०\% naturale) e quella non mi dà affatto noia... per fortuna ho il seno piccolissimo [...] (commento di sweetheart93 al forum gravidanza.alfemminile.com, $\mathrm{r} / 8 / 20 \mathrm{r})$ ).

Su quotidiani e riviste di moda, a parte l'attestazione su "Libero" del 20II, la parola comincia a comparire in alcuni articoli del 2015:

Abbiamo ricevuto molti commenti positivi: la gente sembra sorpresa perché nonostante sia un bralette (reggiseno senza coppe 
preformate) è un modello che offre sostegno e contenimento. E questa è una novità per un reggiseno non sportivo. LC: Ok, di solito il bralette si indossa per circa to minuti prima di andare a letto per poi finire immancabilmente sul pavimento. MR: Guarda li ho provati. Io porto una 34 o una 36D (corrispondenti a 2 e $3 \mathrm{D}$ italiane), quindi le mie proporzioni sono già abbastanza insolite. Indossare un bralette era come indossare una fascia per capelli (Lauren Chan, Land of Women by McKenzie Raley: la modella McKenzie Raley Lancia la linea di lingerie Land of women, vogue.it, 26/1/2015).

Le soluzioni in atto posso [sic] risultare molte e tutte molto utili come vestire canotte aderenti magari realizzate con tessuti foderati, oppure bralette ovvero 'evoluzione dei reggiseni sportivi che possono ricordare piccoli bikini morbidi o top comodi (Alma Zoe Sebastiani, Seno grosso: indossare abiti e top senza reggiseno e senza imbarazzo, ilgiornale.it, I4/6/2015).

Le attestazioni crescono fortemente nel corso del 2016 tanto che si parla di bralette in molte testate di moda online, in blog molto conosciuti come quello di Clio Zammatteo (meglio nota come Cliomakeup), fino a testate giornalistiche come "il Post" e "La Repubblica":

Il bralette è, come vi anticipavamo, un indumento che sta a metà strada tra un reggiseno, un top e un sotto giacca. Non ha imbottitura e neanche ferretti, il tessuto utilizzato è spesso il pizzo [...]. Alcuni modelli risalenti ai primi anni 'zo possono essere definiti bralette anche se a quel tempo venivano comunemente chiamati "reggipetti" (Team Clio, Super Sexy Con Il Bralette: Ecco I Nuovi Reggiseni/Top Che Hanno Conquistato Le Star E Non Solo!, blog.cliomakeup.com, 28/II/2016).

I bralette sono reggiseni senza imbottitura, coppa o ferretto sostenitivo, ma diversi da quelli sportivi perché non comprimono il seno. I bralette esistono da prima che ci fosse una parola apposita per indicarli (i primi reggiseni, prodotti negli anni Venti e Trenta, avevano le stesse caratteristiche), ma ultimamente vanno di moda, anche in Italia. ([s.f.], Sono questi $i$ reggiseni che metteremo nei prossimi anni?, ilpost.it, 16/5/2016).

I bralette sono i reggiseni- top senza supporto ora di gran moda e destinati, ovviamente, alle meno formose (Serena Tibaldi, Ma le donne non sono piu volubili, repubblica.it, I6/7/2016).

Nel 2017 si ha una crescente popolarità del prodotto a cui si associa un notevole incremento d'impiego della parola bralette, come si può notare dalle attestazioni su Google e Twitter:

\begin{tabular}{|c|c|c|c|c|c|c|}
\hline & 2013 & 2014 & 2015 & 2016 & 2017 & 2018 \\
\hline Google (p.i.) & I.050 & I.560 & 3.110 & 6.580 & 16.200 & 3 I.400 \\
\hline Twitter & 3 & 4 & I5 & I7 & 62 & 46 \\
\hline
\end{tabular}

Il successo del 2017 si deve alla fortuna di una campagna pubblicitaria intrapresa da una famosa e popolare casa di moda intima che sponsorizza, con una bellissima modella, il reggiseno bralette, non solo sulle riviste patinate ma anche in tv e soprattutto attraverso una serie di cartelloni pubblicitari. I commenti a proposito della perfetta bellezza della modella e del suo reggiseno si moltiplicano su forum, blog e social network, tanto che il modello bralette diventa un punto di riferimento per la moda intima. Il successo della pubblicità è talmente forte da diventare un vero e proprio caso mediatico e da scatenare aspre polemiche da parte di gruppi femministi:

Ma, come avrete capito, questo non è un post per la categoria "Economia". Guardando Irina Shayk in bralette (e non venitemi a dire che non sapete cosa sia una bralette!) giuro che ho pensato, nellordine: I) Perbacco, che graziosa fanciulla! 2) Ohibò, che ottima manifattura! 3) Ma codesta réclame a chi sarebbe diretta, esattamente? [...] La domanda di cui al punto 3 è ovviamente retorica, almeno nel senso che i meccanismi pubblicitari sono abbastanza noti anche al grande pubblico e io non voglio parlare neppure di pubblicità. La mia personale conclusione, affinché non ci siano equivoci, è molto semplice: no alla volgarizzazione e mercificazione sessista del corpo della donna, e divieto di fare campagne allusive (Claudio Bezzi, Per chi sono realmente fatti i manifesti di Intimissimi. O: la questione di genere è solo una questione di tette?, ilsaltodirodi.com, I7/5/2017).

Lei è Irina Shayk, stratosferica modella russa, scelta [...] per pubblicizzare il nuovo reggiseno-cult, una bralette' in pizzo che ha già conquistato la platea femminile. E se è il capo a funzionare, per estetica e per caratteristiche sartoriali, anche i manifesti hanno avuto il loro peso (Helga Marsala, La pubblicità di Intimissimi indigna le femministe. Basta stereotipi. La bellezza diventa tabu'?, artribune.com, 29/5/2017).

Nel corso del 2017 agli articoli di moda sulle testate giornalistiche nazionali come "La Stampa", "Il Messaggero", "Libero", "Il Giornale" si aggiungono articoli sulle riviste femminili in rete come "Grazia", "Elle", "Donna Moderna", "Tu Style" ecc.

Il successo del capo intimo e la conseguente crescita d'impiego della parola bralette non si arrestano allo scadere 
della citata campagna pubblicitaria: nel 2018, pur registrando una decrescita delle occorrenze su Twitter, la parola ricorre negli articoli di moda di quasi tutti i quotidiani nazionali, coinvolgendo anche "Il Corriere della Sera" e "Il Fatto Quotidiano." Dalla tabella precedente, si evince una crescita d'uso per il 20I8, che viene confermata anche per il 2019: basti pensare che il I3/12/2018 bralette nelle pagine in italiano di Google aveva 867.000 risultati, il 22/I/2019 invece registra ben 1.340 .000 risultati.

Una questione non appianata è rappresentata dal genere grammaticale che viene associato al termine in questione. Infatti c'è una certa discrasia tra i primi testi in cui compare bralette e i testi più recenti. Come si nota dagli esempi citati per il 2016 (che considereremo orientativamente l'anno d'affermazione della parola in italiano), il genere associato a bralette è fondamentalmente il maschile, salvo alcuni sporadici casi rappresentanti una piccola e poco significativa percentuale. Su Twitter, nel 2016, I7 tweet presentano la parola bralette, di cui solo 9 esplicitano il genere grammaticale. Di questi, 4 presentano bralette al femminile e 5 al maschile, confermando solo per poco la tendenza che si registrava per le attestazioni del 20I6. Il rapporto si ribalta nel 20I7, l'anno del successo del reggiseno: dei 62 tweet che presentano bralette, 4I esplicitano il genere grammaticale, di cui zo al femminile e solamente ir al maschile. La tendenza ad associare il femminile viene confermata per tutto il 2017 fino ai giorni nostri dalle occorrenze sui quotidiani, riviste e blog nonché da quelle nei libri:

Vedo Jordan dentro, con indosso dei pantaloncini inguinali e una bralette (Erin Watt, Paper palace. The Royals 3, traduz. di Elena Paganelli, Milano, Sperling \& Kupfer, 2017).

Le spalline devono essere sottili e appena divergenti, non possono superare in spessore la fascia orizzontale per la chiusura [...] Le bralette, ormai ricercatissime, non fanno eccezione (Alberto Caputo, Alice Natoli, Tienilo stretto. Segreti per donne irresistibili, Milano, Mondadori, 2018).

Infatti dalla seguente tabella che si riferisce alle ricerche effettuate sulle pagine in italiano di Google il 22/I/2019, si evince che il genere grammaticale oggi prevalente per bralette è il femminile:

\begin{tabular}{|l|l|l|l|}
\hline \multicolumn{2}{|c|}{ Maschile } & \multicolumn{2}{c|}{ Femminile } \\
\hline "il bralette" & $7 . \mathrm{IIO}$ & "la bralette" & I4.600 \\
\hline "i bralette" & 2.370 & "le bralette" & 707 \\
\hline "un bralette" & I.250 & "una bralette" & 6.050 \\
\hline "dei bralette" & I22 & "delle bralette" & I0.200 \\
\hline "del bralette" & I.590 & "della bralette" & 2.140 \\
\hline TOT. & I2.442 & TOT. & 33.697 \\
\hline
\end{tabular}

Nonostante questa sia la generale tendenza, sui quotidiani continuano a non mancare esempi in cui si preferisce usare il maschile:

I bralette presi in prestito dal cassetto della lingerie diventano il match perfetto di slip micro a vita alta e non [...]. (Laura Bolasco, A vita alta o interi (rigorosamente rossi): ecco i costumi dell'estate 2018, ilmessaggero.it, 27/4/2018).

La difficoltà nell'associazione del genere grammaticale si deve anche al fatto che giocano motivazioni differenti a favore ora del maschile ora del femminile (per l'assegnazione del genere ai forestierismi si veda la risposta di Raffaella Setti). Ad esempio dietro l'uso del maschile si può presupporre un implicito riferimento a il reggiseno bralette o a il modello bralette oppure a il bra. Per quanto riguarda il femminile, che si sta affermando, il suffisso -ette/-et riconduce la parola a una serie di francesismi presenti nel lessico italiano dell'abbigliamento che presentano tutti, il genere femminile: la pochette, la salopette, la longuette, la paillette. Inoltre si potrebbe essere innescato un riferimento implicito a termini francesi che indicano la biancheria intima di classe come la lingerie, la guepière e la stessa brassiére: ciò farebbe di bralette un termine percepito in italiano più come pseudofrancesismo che come anglicismo, quale invece è effettivamente (con possibili riflessi anche nella pronuncia). Si potrebbe anche pensare che abbia contribuito alla diffusione del femminile l'associazione di suono con bretelle (francesismo anche questo), che nel reggiseno in questione sono rappresentate dai caratteristici triangoli di pizzo che coprono il seno. Comunque se questa tendenza fosse stata veramente così forte, avremmo potuto riscontrare la forma singolare braletta, che invece non ha occorrenze significative sul web e si può considerare forma non attestata. La tendenza che l'uso sta confermando è quella di usarebralette al femminile, soprattutto dopo la campagna pubblicitaria del 2017, ma l'affermazione di tale genere potrà essere comprovata soltanto da un'osservazione a distanza di tempo. Nonostante sia poco significativa in termini quantitativi, va segnalata la variante bralet, 
inserita, come si è visto sopra, anche nell'OED; il 26/2/2019 nelle pagine in italiano di Google se ne registrano ben IIo.0oo risultati. Tuttavia, a fronte di i milione e 300 mila della forma bralette, bralet si può considerare una variante minoritaria e trascurabile. Infine bisogna considerare, accanto al genere grammaticale, anche il numero: bralette non è un nome che al plurale può indicare un singolo oggetto come può avvenire per le mutande (cioè 'un paio di mutande'), i pantaloni (cioè 'un paio di pantaloni'). Nonostante la forma della parola che terminando in -e potrebbe alludere a un plurale femminile, quando si usa bralette preceduto dall'articolo plurale (che sia maschile o femminile), il referente rimane plurale: le bralette e $i$ bralette indicano un insieme di reggiseni di questo tipo mentre per indicare il singolo oggetto si usa la bralette e il bralette.

\section{Cita come:}

Miriam Di Carlo, Vedo non vedo la (il) bralette, "Italiano digitale", 2019, VIII, 2019/1 (gennaiomarzo) , pp. 65-68.

DOI: $10.35948 / 2532-9006 / 2019.3088$

\section{Copyright 2019 Accademia della Crusca}

Pubblicato con licenza creative commons CC BY-NC-ND 